\title{
TRACES OF $C^{*}$-ALGEBRAS OF CONNECTED SOLVABLE GROUPS
}

\author{
INGRID BELTIŢĂ AND DANIEL BELTIŢĂ
}

\begin{abstract}
We give an explicit description of the tracial state simplex of the $C^{*}$-algebra $C^{*}(G)$ of an arbitrary connected, second countable, locally compact, solvable group $G$. We show that every tracial state of $C^{*}(G)$ lifts from a tracial state of the $C^{*}$-algebra of the abelianized group, and the intersection of the kernels of all the tracial states of $C^{*}(G)$ is a proper ideal unless $G$ is abelian. As a consequence, the $C^{*}$-algebra of a connected solvable nonabelian Lie group cannot embed into a simple unital AF-algebra.
\end{abstract}

\section{INTRODUCTION}

If $G$ is a locally compact group, then any of its Haar measures leads via integration to a multiplicative linear functional on $C^{*}(G)$, which is in particular a tracial state. Moreover, the following assertions are equivalent:

- $G$ is amenable;

- the reduced group $C^{*}$-algebra $C_{r}^{*}(G)$ is nuclear and admits a tracial state.

See [Ng15, Th. 8] and also [FSW17, Cor. 3.3] and [KR17. There arises the natural question of finding an explicit description of the set of all tracial states of group $C^{*}$-algebras of amenable locally compact groups. In this paper we answer that question for connected, second countable, locally compact, solvable groups, which includes all connected solvable Lie groups (Theorem 2.8(iii)). To this end we prove that the extreme points of the tracial simplex of group $C^{*}$-algebras correspond to the 1-dimensional group representations (Proposition 2.7). This solves the above problem via the Kreln-Milman theorem.

As an application, we show that if the $C^{*}$-algebra of such a group admits a faithful tracial state, then the group under consideration is necessarily abelian (Corollary [2.9). This implies that the $C^{*}$-algebra of a connected solvable nonabelian Lie group never embeds into a simple unital AF-algebra (Corollary 2.10). In addition to our earlier examples of amenable connected Lie groups whose group $C^{*}$-algebras are not AF-embeddable BB18, this is still another aspect of AF-embeddability theory of group $C^{*}$-algebras which points out the sharp contrast between the behaviours of the $C^{*}$-algebras of discrete countable groups and connected Lie groups. We recall that the $C^{*}$-algebra of every amenable discrete countable group embeds into a simple unital AF-algebra, namely, the universal UHF-algebra [Sc20, Th. B].

Key words and phrases. solvable group; solvable Lie group; group $C^{*}$-algebra; trace.

2020 Mathematics Subject Classification. Primary 22D25; Secondary 46L30. 


\section{MAIN RESUlT}

Notation 2.1. For an arbitrary $C^{*}$-algebra $A$ with its topological dual space $A^{*}$ we will use the following notation:

- $A_{+}^{*}:=\left\{\varphi \in A^{*} \mid 0 \leq \varphi\right\}$;

- $A_{+, \mathrm{tr}}^{*}:=\left\{\varphi \in A_{+}^{*} \mid(\forall a, b \in A) \varphi(a b)=\varphi(b a)\right\}$

- $T(A):=\left\{\tau \in A_{+, \text {tr }}^{*} \mid\|\tau\|=1\right\}, T_{\leq 1}(A):=\left\{\tau \in A_{+, \text {tr }}^{*} \mid\|\tau\| \leq 1\right\}$;

- $\operatorname{ext} S:=\{\chi \in S \mid \chi$ is an extreme point of $S\}$ for any subset $S \subseteq A^{*}$;

- $\overline{\mathrm{co}} S$ stands for the weak*-closure of the convex hull of any subset $S \subseteq A^{*}$.

We also note the equality

$$
T_{\leq 1}(A)=\{t \varphi \mid t \in[0,1], \varphi \in T(A)\} .
$$

The elements of $T(A)$ are called tracial states of $A$, and the sets $T(A) \subseteq T_{\leq 1}(A)$ are regarded as a topological subspaces of $A^{*}$ with respect to the weak ${ }^{*}$ topology of $A^{*}$. For every $\tau \in \operatorname{ext} T_{\leq 1}(A)$ with $\|\tau\| \neq 0$ we necessarily have $\|\tau\|=1$, hence $\tau \in T(A)$, and then $\tau \in T(A) \cap \operatorname{ext} T_{\leq 1}(A) \subseteq \operatorname{ext} T(A)$. Thus $\operatorname{ext}\left(T_{\leq 1}(A)\right) \subseteq$ $\{0\} \cup \operatorname{ext}(T(A)) \subseteq T_{\leq 1}(A)$ hence, taking $\overline{c o}(\cdot)$ in these inclusions, we obtain

$$
T_{\leq 1}(A)=\overline{\mathrm{co}}(\{0\} \cup \operatorname{ext} T(A))
$$

since the set $T_{\leq 1}(A)$ is compact and convex, therefore $T_{\leq 1}(A)=\overline{\operatorname{co}}\left(\operatorname{ext} T_{\leq 1}(A)\right)$ by the Kreln-Milman theorem. (In fact, by a slightly more elaborate argument, $\operatorname{ext} T_{\leq 1}(A)=\{0\} \cup \operatorname{ext} T(A)$ by [Di64, Prop. 6.8.7(ii)], since the points of $\operatorname{ext} T(A)$ are the finite characters of norm 1 in the sense of [Di64, 6.7.1].)

We also note for later use in the proof of Theorem 2.8(1) that for any subset $S \subseteq A^{*}$ we have

$$
\bigcap_{\varphi \in \overline{\operatorname{co}}(\{0\} \cup S)} \operatorname{Ker} \varphi=\bigcap_{\varphi \in S} \operatorname{Ker} \varphi=\bigcap_{t \in[0,1]} \bigcap_{\varphi \in S} \operatorname{Ker}(t \varphi)=\bigcap_{\varphi \in \cup_{t \in[0,1]} t S} \operatorname{Ker} \varphi
$$

For any $\varphi \in A_{+}^{*}$ we denote by $\left(\pi_{\varphi}, \mathcal{H}_{\varphi}, \xi_{\varphi}\right)$ the output of its corresponding GNS construction; thus $\pi_{\varphi}: A \rightarrow \mathcal{B}\left(\mathcal{H}_{\varphi}\right)$ is a $*$-representation with a cyclic vector $\xi_{\varphi} \in \mathcal{H}_{\varphi}$ satisfying

$$
(\forall a \in A) \quad \varphi(a)=\left(\pi_{\varphi}(a) \xi_{\varphi} \mid \xi_{\varphi}\right) .
$$

The notation $J \unlhd A$ indicates that $J$ is a closed two-sided ideal of the $C^{*}$-algebra $A$.

We prove the following lemma for completeness, although its assertions are mostly known, as indicated in Remark 2.3 below.

Lemma 2.2. For every $\varphi \in A_{+, \text {tr }}^{*}$ the following assertions hold:

(i) One has $A^{+} \cap \operatorname{Ker} \varphi=A^{+} \cap \operatorname{Ker} \pi_{\varphi}$.

(ii) The functional $\tau_{\varphi}: \pi_{\varphi}(A)^{\prime \prime} \rightarrow \mathbb{C}, \tau_{\varphi}(T):=\left(T \xi_{\varphi} \mid \xi_{\varphi}\right)$, is a faithful normal tracial positive functional hence the von Neumann algebra $\pi_{\varphi}(A)^{\prime \prime}$ is finite.

(iii) If $\varphi \in \operatorname{ext} T(A)$, then the von Neumann algebra $\pi_{\varphi}(A)^{\prime \prime}$ is a factor.

Proof. (ii) It folows by [Di64, Cor. 2.4.10] that Ker $\pi_{\varphi}$ is the largest closed two-sided ideal of $A$ contained in $\operatorname{Ker} \varphi$. 
To prove that $A^{+} \cap \operatorname{Ker} \varphi \subseteq \operatorname{Ker} \pi_{\varphi}$ let $a \in A^{+}$arbitrary with $\varphi(a)=0$. For every $b \in A$ one has by the Schwarz inequality $\left|\varphi\left(a^{1 / 2} b\right)\right|^{2} \leq \varphi(a) \varphi\left(b^{*} b\right)=0$, hence $a^{1 / 2} b \in \operatorname{Ker} \varphi$. Then for arbitrary $c \in A$ one has

$$
\left\|\pi_{\varphi}\left(a^{1 / 4}\right) \pi_{\varphi}(c) \xi_{\varphi}\right\|^{2}=\left(\pi_{\varphi}\left(a^{1 / 2}\right) \pi_{\varphi}(c) \xi_{\varphi} \mid \pi_{\varphi}(c) \xi_{\varphi}\right)=\varphi\left(c^{*} a^{1 / 2} c\right)=\varphi\left(a^{1 / 2} c c^{*}\right)=0
$$

by the above remark, with $b=c c^{*}$. Thus $\pi_{\varphi}\left(a^{1 / 4}\right) \pi_{\varphi}(c) \xi_{\varphi}=0$ for all $c \in A$. Since $\pi_{\varphi}(A) \xi_{\varphi}$ is dense in $\mathcal{H}_{\varphi}$, we then obtain $\pi_{\varphi}\left(a^{1 / 4}\right)=0$, hence $\pi_{\varphi}(a)=0$.

(iii) It is clear that $\tau_{\varphi}$ is a normal positive functional. The set $\pi_{\varphi}(A)$ is dense in $\pi_{\varphi}(A)^{\prime \prime}$ with respect to the strong operator topology (by the bicommutant theorem). Therefore, in order to see that $\tau_{\varphi}$ is a tracial functional, it suffices to show that $\left.\tau_{\varphi}\right|_{\pi_{\varphi}(A)}$ is tracial. The later property follows by $\varphi \in A_{+, \text {tr }}^{*}$ since for all $a \in A$ one has $\tau_{\varphi}\left(\pi_{\varphi}(a)\right)=\varphi(a)$ by (2.4).

The fact that $\tau_{\varphi}$ is faithful follows as in the proof of [BB18, Lemma 4.2]: Let $T \in \pi_{\varphi}(A)^{\prime \prime}$ with $\tau_{\varphi}\left(T^{*} T\right)=0$, that is, $T \xi_{\varphi}=0$. Then for every $a \in A$,

$$
\left\|T \pi_{\varphi}(a) \xi_{\varphi}\right\|^{2}=\left(\pi_{\varphi}(a)^{*} T^{*} T \pi_{\varphi}(a) \xi_{\varphi} \mid \xi_{\varphi}\right)=\tau_{\varphi}\left(\pi_{\varphi}(a)^{*} T^{*} T \pi_{\varphi}(a)\right) .
$$

Since $\tau_{\varphi}$ is a tracial state of $\pi_{\varphi}(A)^{\prime \prime}$, it follows that

$$
\left\|T \pi_{\varphi}(a) \xi_{\varphi}\right\|^{2}=\tau_{\varphi}\left(\pi_{\varphi}(a) \pi_{\varphi}(a)^{*} T^{*} T\right)=\left(\pi_{\varphi}(a) \pi_{\varphi}(a)^{*} T^{*} T \xi_{\varphi} \mid \xi_{\varphi}\right)=0,
$$

Thus $T \pi_{\varphi}(a) \xi_{\varphi}=0$ for every $a \in A$, hence $T=0$,

(iii) For every central projection $p=p^{*}=p^{2} \in \pi_{\varphi}(A)^{\prime \prime} \cap \pi_{\varphi}(A)^{\prime}$ we define

$$
\varphi_{p}(\cdot):=\left(\pi_{\varphi}(\cdot) p \xi_{\varphi} \mid p \xi_{\varphi}\right)=\left(\pi_{\varphi}(\cdot) \xi_{\varphi} \mid p \xi_{\varphi}\right)
$$

so that $\varphi=\varphi_{p}+\varphi_{1-p}$ and $\varphi_{p}, \varphi_{1-p} \in A_{+}^{*}$, where $\mathbf{1} \in \mathcal{B}\left(\mathcal{H}_{\varphi}\right)$ is the identity operator. Moreover, since $p \in \pi_{\varphi}(A)^{\prime \prime}$ and $\pi_{\varphi}(A)$ is dense in $\pi_{\varphi}(A)^{\prime \prime}$ with respect to the strong operator topology, there exists a net $\left\{c_{i}\right\}_{i \in I}$ in $A$ with $p=\lim _{i \in I} \pi_{\varphi}\left(c_{i}\right)$ in the strong operator topology in $\mathcal{B}\left(\mathcal{H}_{\varphi}\right)$. Then for arbitrary $a, b \in A$ we obtain

$$
\begin{aligned}
\varphi_{p}(a b) & =\left(\pi_{\varphi}(a b) p \xi_{\varphi} \mid \xi_{\varphi}\right)=\left(\pi_{\varphi}(a) p \pi_{\varphi}(b) \xi_{\varphi} \mid \xi_{\varphi}\right)=\lim _{i \in I}\left(\pi_{\varphi}\left(a c_{i} b\right) \xi_{\varphi} \mid \xi_{\varphi}\right) \\
& =\lim _{i \in I} \varphi\left(a c_{i} b\right)=\lim _{i \in I} \varphi\left(c_{i} b a\right)=\lim _{i \in I}\left(\pi_{\varphi}\left(c_{i} b a\right) \xi_{\varphi} \mid \xi_{\varphi}\right)=\left(p \pi_{\varphi}(b a) \xi_{\varphi} \mid \xi_{\varphi}\right) \\
& =\varphi_{p}(b a),
\end{aligned}
$$

by (2.4) and the fact that $\varphi$ is a tracial functional. Thus $\varphi_{p} \in A_{+, \text {tr }}^{*}$ and similarly $\varphi_{1-p} \in A_{+, \text {tr }}^{*}$. If $p \xi_{\varphi} \neq 0$ and $(\mathbf{1}-p) \xi_{\varphi} \neq 0$, then $\psi_{0}:=\frac{1}{\left\|p \xi_{\varphi}\right\|^{2}} \varphi_{p} \in T(A)$ and $\psi_{1}:=\frac{1}{\left\|(\mathbf{1}-p) \xi_{\varphi}\right\|^{2}} \varphi_{\mathbf{1}-p} \in T(A)$ and moreover $\varphi=\left\|p \xi_{\varphi}\right\|^{2} \psi_{0}+\left\|(\mathbf{1}-p) \xi_{\varphi}\right\|^{2} \psi_{1}$, where $\left\|p \xi_{\varphi}\right\|^{2}+\left\|(\mathbf{1}-p) \xi_{\varphi}\right\|^{2}=\left\|\xi_{\varphi}\right\|^{2}=\|\varphi\|=1$, hence we obtain a contradiction with the hypothesis $\varphi \in \operatorname{ext} T(A)$.

Therefore one must have either $p \xi_{\varphi}=0$ or $(\mathbf{1}-p) \xi_{\varphi}=0$, that is, either $p \xi_{\varphi}=\xi_{\varphi}$ or $p \xi_{\varphi}=0$. Then, using the fact that $\pi_{\varphi}(A) \xi_{\varphi}$ is dense in $\mathcal{H}_{\varphi}$ and $p \in \pi_{\varphi}(A)^{\prime}$, we obtain either $p=\mathbf{1}$ or $p=0$. Since $p$ is an arbitrary orthogonal projection in the center of $\pi_{\varphi}(A)^{\prime \prime}$, it follows that $\pi_{\varphi}(A)^{\prime \prime}$ is a factor.

Remark 2.3. Lemma 2.2(iii) can also be derived from [Di64, Prop. 6.8.3]. As noted above, the points of ext $T(A)$ are the finite characters of norm 1, therefore the conclusion of Lemma2.2(iii) can be obtained by Di64, Th. 6.7.3]. We also recall that the finite characters of norm 1 correspond bijectively to the quasi-equivalence classes of nontrivial finite-factor representations by [Di64, Cor. 6.8.6]. 
For an arbitrary locally compact group $G$ we denote

$$
G^{(1)}:=\text { the closed subgroup generated by }\left\{g h g^{-1} h^{-1} \mid g, h \in G\right\} \text {. }
$$

Then $G^{(1)}$ is a closed normal subgroup of $G$ and, by [BkHa19, Prop. 8.C.8], there exists a natural surjective $*$-morphism

$$
\theta: C^{*}(G) \rightarrow C^{*}\left(G / G^{(1)}\right)
$$

Hence, denoting

$$
\mathcal{J}_{G}:=\operatorname{Ker} \theta,
$$

we obtain the short exact sequence of $C^{*}$-algebras

$$
0 \rightarrow \mathcal{J}_{G} \hookrightarrow C^{*}(G) \stackrel{\theta}{\longrightarrow} C^{*}\left(G / G^{(1)}\right) \rightarrow 0 .
$$

Lemma 2.4. We have that

$$
\mathcal{J}_{G}=\{0\} \Longleftrightarrow G \text { is commutative. }
$$

Proof. If the group $G$ is abelian then $G^{(1)}=\{\mathbf{1}\}$ and $\theta$ is a *-isomorphism, hence $\mathcal{J}_{G}=\{0\}$. Conversely, if $\mathcal{J}_{G}=\{0\}$, then the short exact sequence (2.7) shows that $\theta: C^{*}(G) \rightarrow C^{*}\left(G / G^{(1)}\right)$ is a $*$-isomorphism. Since the quotient group $G / G^{(1)}$ is abelian, its $C^{*}$-algebra is commutative. It then follows that $C^{*}(G)$ is commutative, which in turn implies that its dense $*$-subalgebra $L^{1}(G)$ is commutative, and this further implies that the group $G$ is abelian. (See [Ri60, A.3.1, page 321] and also [HR63, Th. (20.24)] for a more general result.)

We denote by $P_{1}(G) \subseteq L^{\infty}(G)$ the set of all positive-definite functions $f: G \rightarrow \mathbb{C}$ with $f(\mathbf{1})=1$, and let

$$
P_{1, c}(G):=\left\{f \in P_{1}(G) \mid(\forall x, y \in G) \quad f\left(x y x^{-1}\right)=f(y)\right\}
$$

be the set of all central positive-definite functions.

For every $f \in P_{1}(G)$, let $\varphi_{f}: C^{*}(G) \rightarrow \mathbb{C}$ be its corresponding state of $C^{*}(G)$. By [Di64, Th. 13.5.2], the affine mapping

$$
\Phi: P_{1}(G) \rightarrow S\left(C^{*}(G)\right), \quad f \mapsto \varphi_{f}
$$

is a homeomorphism when $P_{1}(G)$ is endowed with the topology of uniform convergence on the compact subsets of $G$, while the state space $S\left(C^{*}(G)\right)$ is endowed with its weak*-topology. It is also well-known that

$$
\Phi\left(P_{1, c}(G)\right)=T\left(C^{*}(G)\right) .
$$

(See e.g., [Di64, 18.1.3 and proof of Prop. 17.3.1] and [FSW17, §1.1].)

Remark 2.5. In the above setting, we note for later use that $\operatorname{Hom}(G, \mathbb{T}) \subseteq P_{1, c}(G)$. Thus if $\chi \in \operatorname{Hom}(G, \mathbb{T})$ and if we denote by $\widetilde{\chi}: C^{*}(G) \rightarrow \mathbb{C}$ the $*$-representation obtained by integrating the unitary representation $\chi$, then for every $g \in L^{1}(G) \subseteq$ $C^{*}(G)$ we have $\widetilde{\chi}(g)=\int_{G} g(x) \chi(x) \mathrm{d} x=\varphi_{\chi}(g)$, hence

$$
\varphi_{\chi}=\tilde{\chi} \text { for every } \chi \in \operatorname{Hom}(G, \mathbb{T}) \text {. }
$$

Lemma 2.6. Let $G$ be a connected, locally compact group and $A:=C^{*}(G)$. If $\varphi \in \operatorname{ext} T(A)$, then there exist a positive integer $n \geq 1$ such that $\pi_{\varphi}$ is weakly equivalent to an irreducible representation of $A$ on an $n$-dimensional Hilbert space. If moreover the group $G$ is solvable, then $n=\operatorname{dim} \mathcal{H}_{\varphi}=1$ and there exists $\chi \in$ $\operatorname{Hom}(G, \mathbb{T})$ with $\pi_{\varphi}=\tilde{\chi}$ 
Proof. It follows by Lemma 2.2 that $\pi_{\varphi}(A)^{\prime \prime}$ is a finite factor. On the other hand, since the representation $\pi_{\varphi}: A=C^{*}(G) \rightarrow \mathcal{B}\left(\mathcal{H}_{\varphi}\right)$ is cyclic, hence nondegenerate, it corresponds to a unitary representation of $G$. Therefore, since the group $G$ is connected, it follows by [KS52, Th. 1] that $\pi_{\varphi}$ has no type $\mathrm{II}_{1}$ direct summand. Thus the finite factor $\pi_{\varphi}(A)^{\prime \prime}$ is not type $\mathrm{II}_{1}$, and then it is type $\mathrm{I}_{n}$ for some positive integer $n$, that is, one has a $*$-isomorphism $\pi_{\varphi}(A)^{\prime \prime} \simeq M_{n}(\mathbb{C})$. Since $\pi_{\varphi}(A)$ is dense in $\pi_{\varphi}(A)^{\prime \prime}$ with respect to the strong operator topology, it follows that $\pi_{\varphi}(A) \simeq M_{n}(\mathbb{C})$.

Since $\pi_{\varphi}$ is a factor representation, there exists an irreducible *-representation $\chi: A=C^{*}(G) \rightarrow \mathcal{B}\left(\mathcal{H}_{\chi}\right)$ (see [Di64, 3.9.1(c) and 5.7.6((b),(d))]) with Ker $\pi_{\varphi}=$ Ker $\chi$. One then has $*$-isomorphisms

$$
\chi(A) \simeq A / \operatorname{Ker} \chi=A / \operatorname{Ker} \pi_{\varphi} \simeq \pi_{\varphi}(A) \simeq M_{n}(\mathbb{C})
$$

and it then follows that $\operatorname{dim} \mathcal{H}_{\chi}=n$. Moreover, the irreducible $*$-representation $\chi$ is obtained by integrating a certain unitary irreducible representation of $G$, denoted also as $\chi: G \rightarrow U(n) \subseteq M_{n}(\mathbb{C})$.

If moreover the group $G$ is solvable, we obtain $n=1$ by Sophus Lie's classical theorem. (See e.g., [Se06, Part I, Ch. V, §5].) Therefore $\pi_{\varphi}(A)=\pi_{\varphi}(A)^{\prime \prime}=\mathbb{C} \mathbf{1}$. Then, since $\xi_{\varphi} \in \mathcal{H}_{\varphi}$ is a cyclic vector for the representation $\pi_{\varphi}$ we obtain $\mathcal{H}_{\varphi}=$ $\mathbb{C} \xi_{\varphi}$. Thus $\operatorname{dim} \mathcal{H}_{\varphi}=1$, hence $\chi \in \operatorname{Hom}(G, \mathbb{T})$, and this completes the proof.

Proposition 2.7. If $G$ is a connected, locally compact, solvable group, then the map $\left.\Phi\right|_{\operatorname{Hom}(G, \mathbb{T})}: \operatorname{Hom}(G, \mathbb{T}) \rightarrow \operatorname{ext} T\left(C^{*}(G)\right), \chi \mapsto \varphi_{\chi}$ is a homeomorphism.

Proof. Use Lemma 2.6 and (2.9)-(2.10).

In the statement of the next theorem, we use the notation in (2.5) and (2.6).

Theorem 2.8. Let $G$ be a connected, locally compact, solvable group. Then the following assertions hold:

(i) The closed two-sided ideal $\mathcal{J}_{G}$ is the interesection of all kernels of tracial states of $C^{*}(G)$, that is,

$$
\mathcal{J}_{G}=\bigcap_{\varphi \in T\left(C^{*}(G)\right)} \operatorname{Ker} \varphi .
$$

(ii) $\mathcal{J}_{G}$ is equal to the closed linear span of any of the sets

$$
\left\{a b-b a \mid a, b \in C^{*}(G)\right\} \quad \text { and } \quad\left\{a \in C^{*}(G) \mid a^{2}=0\right\} .
$$

(iii) The mapping $T\left(C^{*}\left(G / G^{(1)}\right)\right) \rightarrow T\left(C^{*}(G)\right), \tau \mapsto \tau \circ \theta$, is bijective.

Proof. (ii) We actually prove the following equality:

$$
\mathcal{J}_{G}=\bigcap_{\chi \in \operatorname{Hom}(G, \mathbb{T})} \operatorname{Ker} \varphi_{\chi}=\bigcap_{\varphi \in T\left(C^{*}(G)\right)} \operatorname{Ker} \varphi
$$


It follows by Proposition 2.7 and the consequence of the Kreun-Milman theorem (2.2) along with (2.3) and (2.1) that

$$
\begin{aligned}
\bigcap_{\chi \in \operatorname{Hom}(G, \mathbb{T})} \operatorname{Ker} \varphi_{\chi} & =\bigcap_{\varphi \in \operatorname{ext} T\left(C^{*}(G)\right)} \operatorname{Ker} \varphi \\
& =\bigcap_{\varphi \in \overline{\operatorname{co}}\left(\{0\} \cup \operatorname{ext} T\left(C^{*}(G)\right)\right)} \operatorname{Ker} \varphi \\
& =\bigcap_{\varphi \in T \leq 1} \operatorname{Ker} \varphi \\
& =\bigcap_{\varphi \in T\left(C^{*}(G)\right)} \operatorname{Ker} \varphi,
\end{aligned}
$$

hence the second equality in (2.12) holds true.

We now prove the first equality in (2.12). Let $q: G \rightarrow G / G^{(1)}$ be the canonical quotient map. Then, since the group $G / G^{(1)}$ is abelian, the map

$$
q^{*}: \widehat{G / G^{(1)}} \rightarrow \operatorname{Hom}(G, \mathbb{T}), \quad \omega \mapsto \omega \circ q
$$

is bijective and is actually a homeomorphism. Using the canonical homeomorphism $\widehat{G} \simeq \widehat{C^{*}(G)}$ along with the short exact sequence (2.7), we then obtain

$$
\mathcal{J}_{G}=\bigcap_{\omega \in \widehat{G / G^{(1)}}} \operatorname{Ker} \widetilde{\omega \circ q}=\bigcap_{\chi \in \operatorname{Hom}(G, \mathbb{T})} \operatorname{Ker} \tilde{\chi}=\bigcap_{\chi \in \operatorname{Hom}(G, \mathbb{T})} \operatorname{Ker} \varphi_{\chi}
$$

where the later equality follows by (2.11).

(iii) Let us denote by $A_{0}$ the set of all norm-convergent series $\sum_{n>1}\left(a_{n} a_{n}^{*}-a_{n}^{*} a_{n}\right)$ for $a_{1}, a_{2}, \ldots \in A:=C^{*}(G)$. If we denote by $A_{00}$ the closed linear span of the set $\left\{a b-b a \mid a, b \in C^{*}(G)\right\}$, then it is clear that

$$
A_{0}+\mathrm{i} A_{0} \subseteq A_{00} \subseteq \bigcap_{\varphi \in T(A)} \operatorname{Ker} \varphi=A_{0}+\mathrm{i} A_{0}
$$

where the latter equality follows by [CP79, Thms. 2.6 and 2.9]. Therefore, the above inclusions are actually equalities and then, using (2.12), we obtain $A_{00}=\mathcal{J}_{G}$. On the other hand, $A_{00}$ coincides with the closed linear span of $\left\{a \in C^{*}(G) \mid a^{2}=0\right\}$ by [AEVBS, Prop. 2.2].

(iiii) As $\theta$ is a surjective $*$-morphism, the mapping $T\left(C^{*}\left(G / G^{(1)}\right)\right) \rightarrow T\left(C^{*}(G)\right)$, $\tau \mapsto \tau \circ \theta$, is well defined and injective. To prove that this mapping is surjective, let $\varphi \in T\left(C^{*}(G)\right)$ arbitrary. Then $\operatorname{Ker} \theta=\mathcal{J}_{G} \subseteq \operatorname{Ker} \varphi$ by (ii), hence the functional $\tau: C^{*}\left(G / G^{(1)}\right) \rightarrow \mathbb{C}, \theta(a) \mapsto \varphi(a)$, is well defined, and moreover $\tau \circ \theta=\varphi$. Since the group $G / G^{(1)}$ is abelian, we directly obtain $\tau \in T\left(C^{*}\left(G / G^{(1)}\right)\right)$, and this completes the proof.

We are now in a position to prove the following generalization of [BB18, Prop. 4.4] to groups that need not be type I.

Corollary 2.9. If $G$ is a connected, second countable, locally compact, solvable group, then there exists a faithful $\varphi \in T\left(C^{*}(G)\right)$ if and only if $G$ is abelian. 
Proof. If $G$ is abelian, then its dual group $\widehat{G}$ is second countable by BkHa19, Prop. A.G.3]. Then we may select a dense sequence $\left\{\chi_{n}\right\}_{n \geq 1}$ in $\widehat{G}$. Denoting by $\delta_{\chi}$ the Dirac measure concentrated at any point $\chi \in \widehat{G}$, it then follows that $\mu:=\sum_{n \geq 1} \frac{1}{2^{n}} \delta_{\chi_{n}}$ is a probability Radon measure on $\widehat{G}:=\operatorname{Hom}(G, \mathbb{T})$ with $\operatorname{supp} \mu=\widehat{G}$. We then define

$$
f: G \rightarrow \mathbb{C}, \quad f(x):=\int_{\widehat{G}} \chi(x) \mathrm{d} \mu(\chi) .
$$

Since $G$ is abelian, it is clear that $f \in P_{1, c}(G)$. On the other hand the Fourier transform gives the $*$-isomorhism $C^{*}(G) \simeq \mathcal{C}_{0}(\widehat{G})$. Then, since supp $\mu=\widehat{G}$, it is straightforward to check that $\varphi_{f} \in T\left(C^{*}(G)\right)$ is faithful.

Conversely, if there exists $\varphi \in T\left(C^{*}(G)\right)$ with $\left(C^{*}(G)\right)^{+} \cap \operatorname{Ker} \varphi=\{0\}$ then, by (2.12), we obtain $\left(C^{*}(G)\right)^{+} \cap \mathcal{J}_{G}=\{0\}$. Since $\mathcal{J}_{G}$ is a $C^{*}$-algebra, this implies $\mathcal{J}_{G}=\{0\}$ since $\mathcal{J}_{G}$ is linearly spanned by its positive cone $\left(C^{*}(G)\right)^{+} \cap \mathcal{J}_{G}$. (See e.g., Di64, 1.5.7(2)].) Then Lemma 2.4 shows that the group $G$ is abelian. Alternatively, $\mathcal{J}_{G}=\{0\}$ implies by Theorem 2.8(1ii) that $C^{*}(G)$ is commutative, and then the group $G$ is abelian as in the proof of Lemma 2.4 .

Corollary 2.10. If $G$ is a connected, second countable, locally compact, solvable group, then the following assertions are equivalent:

(i) $C^{*}(G)$ embeds as a closed $*$-subalgebra of a unital simple AF-algebra.

(ii) $C^{*}(G)$ embeds as a closed $*$-subalgebra of a unital quasidiagonal simple $C^{*}$ algebra.

(iii) $G$ is abelian.

Proof. (ii) $\Rightarrow$ (iii): Clear.

(iii) $\Rightarrow$ (iii): Let us assume that there exists a unital quasidiagonal simple $C^{*}$ algebra $A$ with an embedding $C^{*}(G) \subseteq A$. Since $A$ is unital quasidiagonal, it follows by [B106, Prop. V.4.2.7] that $A$ has a tracial state $\varphi: A \rightarrow \mathbb{C}$. Since $A$ is simple, we have that $\operatorname{Ker} \pi_{\varphi}=\{0\}$. Thus Lemma 2.2(1) implies $A^{+} \cap \operatorname{Ker} \varphi=\{0\}$, that is, $\varphi$ is faithful. Then $\left.\varphi\right|_{C^{*}(G)}$ is a faithful positive tracial functional on $C^{*}(G)$, which is moreover nonzero since the $C^{*}$-algebra $C^{*}(G)$ is generated by its positive cone. Therefore $G$ is abelian by Corollary 2.9.

(iii) $\Rightarrow$ (ii): Since $G$ is abelian, there is a $*$-isomorphism $C^{*}(G) \simeq \mathcal{C}_{0}(\widehat{G})$. Let $\bar{G}$ denote the 1-point compactification of $\widehat{G}$ if $\widehat{G}$ is not compact, and $\bar{G}=\widehat{G}$ otherwise. Since $G$ is second countable, its dual group $\widehat{G}$ is second countable by BkHa19, Prop. A.G.3], hence $\bar{G}$ is a compact metrizable space. Then there exists a continuous surjective mapping $K \rightarrow \bar{G}$, where $K$ is the Cantor set, hence one has embeddings

$$
C^{*}(G) \simeq \mathcal{C}_{0}(\widehat{G}) \hookrightarrow \mathcal{C}(\bar{G}) \hookrightarrow \mathcal{C}(K) .
$$

On the other hand, it is well-known that $\mathcal{C}(K)$ is $*$-isomorphic to the canonical diagonal Cartan subalgebra of the CAR-algebra $M_{2} \infty$. We thus finally obtain an embedding $C^{*}(G) \hookrightarrow M_{2}$, where $M_{2 \infty}$ is a unital simple AF-algebra.

Remark 2.11. The $C^{*}$-algebras of many connected solvable Lie groups are AFembeddable as shown in [BB18] and [BB20, hence Corollary 2.10] shows that such 
embeddings cannot be done into unital simple AF-algebras in the case of nonabelian groups.

Remark 2.12. If $G$ is a connected, separable, locally compact, solvable group, using the notation of [Br06, Def. 3.4.1], one has

$$
T\left(C^{*}(G)\right)=\mathrm{UAT}\left(C^{*}(G)\right)_{\mathrm{LFD}}
$$

that is, every tracial state of $C^{*}(G)$ is uniform locally finite dimensional, and in particular is quasidiagonal. (See [Br06, §3.5].)

In fact, for arbitrary $\varphi \in T\left(C^{*}(G)\right)$, there exists $\tau \in T\left(C^{*}\left(G / G^{(1)}\right)\right)$ with $\varphi=$ $\tau \circ \theta$ by Theorem 2.8(iii). The quotient group $G / G^{(1)}$ is an abelian Lie group, hence $C^{*}\left(G / G^{(1)}\right)$ is type I, and then $\tau \in \operatorname{UAT}\left(C^{*}\left(G / G^{(1)}\right)\right)_{\text {LFD }}$ by [Br06, Cor. 4.4.4]. Now, by [Br06, Prop. 3.5.6], we obtain $\varphi \in \operatorname{UAT}\left(C^{*}(G)\right)_{\text {LFD }}$. This proves the inclusion $\supseteq$ in (2.13), while the converse inclusion is obvious.

However, we recall that there exist connected solvable Lie groups whose $C^{*}$ algebras are not quasidiagonal. (See [BB18, Thm. 2.15].)

Acknowledgment. We wish to thank the Referee for correcting some errors in the first version of our manuscript.

\section{REFERENCES}

[AevBS] J. Alaminos, J. Extremera, A.R. Villena, M. Brešar, Š. Špenko, Commutators and square-zero elements in Banach algebras. Q. J. Math. 67 (2016), no. 1, 1-13.

[BkHa19] B. BEKKA, P. DE LA HARPE, Unitary representations of groups, duals, and characters. Preprint arXiv:1912.07262

[BB18] I. BeltiŢĂ, D. Beltiţă, Quasidiagonality of $C^{*}$-algebras of solvable Lie groups. Integral Equations Operator Theory 90 (2018), no. 1, Art. 5, 21 pp.

[BB20] I. BeLtiţĂ, D. Beltiţ $\breve{A}, \mathrm{AF}$-embeddability for Lie groups with $T_{1}$ primitive ideal spaces. Preprint arXiv: 2004.11010 [math.OA].

[Bl06] B. Blackadar, Operator algebras. Theory of $C^{*}$-algebras and von Neumann algebras. Encyclopaedia of Mathematical Sciences, 122. Operator Algebras and Non-commutative Geometry, III. Springer-Verlag, Berlin, 2006.

[Br06] N.P. Brown, Invariant means and finite representation theory of $C^{*}$-algebras. Mem. Amer. Math. Soc. 184 (2006), no. 865, viii+105 pp.

[CP79] J. Cuntz, G.K. Pedersen, Equivalence and traces on $C^{*}$-algebras. J. Functional Analysis 33 (1979), no. 2, 135-164.

[Di64] J. Dixmier, Les $C^{*}$-algèbres et leurs représentations. Cahiers Scientifiques, Fasc. XXIX Gauthier-Villars \& Cie, Éditeur-Imprimeur, Paris, 1964.

[FSW17] B.E. Forrest, N. Spronk, M. Wiersma, Existence of tracial states on reduced group $C^{*}$-algebras. Preprint arXiv:1706.05354 [math.OA].

[HR63] E. HewitT, K.A. Ross, Abstract harmonic analysis. Vol. I. Die Grundlehren der mathematischen Wissenschaften, Bd. 115. Springer-Verlag, Berlin, 1963.

[KS52] R.V. KADison, I.M. Singer, Some remarks on representations of connected groups. Proc. Natl. Acad. Sci. USA 38 (1952) 419-423.

[KR17] M. Kennedy, S. Raum, Traces on reduced group $C^{*}$-algebras. Bull. Lond. Math. Soc. 49 (2017), no. 6, 988-990.

[Ng15] C.-K. NG, Strictly amenable representations of reduced group $C^{*}$-algebras. Int. Math. Res. Not. IMRN (2015), no. 17, 7853-7860.

[Ri60] C.E. RICKART, General theory of Banach algebras. The University Series in Higher Mathematics D. van Nostrand Co., Inc., Princeton, N.J.-Toronto-London-New York, 1960.

[Sa71] S. SAKAI, $C^{*}$-algebras and $W^{*}$-algebras. Ergebnisse der Mathematik und ihrer Grenzgebiete, Band 60. Springer-Verlag, New York-Heidelberg, 1971. 
[Sc20] Ch. Schafhauser, Subalgebras of simple AF-algebras. Ann. of Math. (2) 192 (2020), no. 2, 309-352.

[Se06] J.-P. SerRe, Lie algebras and Lie groups. Lecture Notes in Mathematics, 1500. Springer-Verlag, Berlin, 2006.

Institute of Mathematics "Simion Stollow" of the Romanian Academy, P.O. Box 1764, Bucharest, Romania

Email address: Ingrid.Beltita@imar.ro, ingrid.beltita@gmail.com

Email address: Daniel.Beltita@imar.ro, beltita@gmail.com 